\title{
Efficiency of Radiolabeling Eggs Before and After Microwave Cooking for Gastric Emptying Scintigraphy Studies
}

\author{
Jena-Lee McKee ${ }^{1}$, Mary Beth Farrell ${ }^{2}$, Kathy Hunt ${ }^{3}$, Vivian Loveless ${ }^{4}$, and Charity Brannen ${ }^{3}$ \\ ${ }^{1}$ West Tennessee Healthcare, Dyersburg Hospital, Dyersburg, Tennessee; ${ }^{2}$ Intersocietal Accreditation Commission, Ellicott City, \\ Maryland; ${ }^{3}$ Baptist College of Health Sciences, Memphis, Tennessee; and ${ }^{4}$ UTHSC College of Pharmacy, Memphis, Tennessee
}

\begin{abstract}
The accuracy and reproducibility of nuclear medicine gastric emptying scintigraphy (GES) require strict adherence to the Society of Nuclear Medicine and Molecular Imaging standardized protocol, which contains precise instructions for meal ingredients and preparation. Previous research demonstrated that many laboratories were using whole eggs in the test meal
\end{abstract} as opposed to the guideline-recommended liquid egg whites and that some laboratories were attempting to radiolabel the egg by adding the radiotracer after cooking. This study aimed to document the labeling efficiency of $99 \mathrm{mT}$-sulfur colloid (SC) added to whole eggs before and after microwave cooking. Methods: Whole eggs were mixed with $99 \mathrm{mTc}-\mathrm{SC}$ before and after microwave cooking. The radiolabeling stability of the eggs was tested after 2 and $4 \mathrm{~h}$ of incubation in gastric fluid simulated using just hydrochloric acid $(\mathrm{HCl})$ and using $\mathrm{HCl}$ with pepsin. Results: The experiment showed that no matter what the testing condition, radiolabeling by adding $99 \mathrm{mTc}-\mathrm{SC}$ to whole eggs before cooking resulted in a significantly higher labeling efficiency than radiolabeling by squirting the $99 \mathrm{mTC}-\mathrm{SC}$ on eggs after cooking. This finding persisted over time, with the precooking method still showing significantly higher radiolabeling at 2 and $4 \mathrm{~h}$ after the egg was placed in the incubation medium for both gastric fluid mediums. For simulated gastric fluid with pepsin at $2 \mathrm{~h}$, the labeling was significantly higher, at $73.3 \%$, when the radiotracer was added before cooking than the $43.3 \%$ when added after cooking $(P<0.001)$. The results of this study further showed that when egg labeling efficiency was tested in $\mathrm{HCl}$ without pepsin, the labeling was less stable than when tested in $\mathrm{HCl}$ with pepsin. In the $\mathrm{HCl}$-only medium, the labeling efficiency decreased significantly between 2 and $4 \mathrm{~h}$ for both radiolabeling methods. Conclusion: The results of this study demonstrated that the addition of ${ }^{99 \mathrm{~m}} \mathrm{Tc}-\mathrm{SC}$ to whole eggs after cooking resulted in considerably inferior binding of the radiotracer to the eggs and that binding deteriorated significantly over time. The study further demonstrated that the results of radiolabeling efficiency varied depending on whether $\mathrm{HCl}$ or $\mathrm{HCl}$ with pepsin was used to simulate gastric fluid. Radiolabeling stability decreased over time when $\mathrm{HCl}$ without pepsin was used. The findings emphasize the criticality of adhering to the standardized meal and preparation, as alternate cooking methods have different radiolabeling efficiencies.

Received Jan. 2, 2019; revision accepted Mar. 18, 2019.

For correspondence or reprints contact: Jena-Lee McKee, West TN Healthcare, Dyersburg Hospital, 541 Erwin Rd., Covington, TN 38019.

E-mail: jenalee.mckee@gmail.com

Published online Apr. 24, 2019.

COPYRIGHT (c) 2019 by the Society of Nuclear Medicine and Molecular Imaging.
Key Words: gastric emptying scintigraphy; radiolabeling stability; guidelines; meal preparation

J Nucl Med Technol 2019; 47:144-148

DOI: 10.2967/jnmt.118.225177

$\mathbf{G}$ astric emptying scintigraphy (GES) is frequently performed in nuclear medicine to evaluate gastric motility (1). GES is accomplished by radiolabeling the solid or liquid component of a meal and then measuring the radioactivity in the stomach over time. It has become the gold standard for measuring the rate of gastric emptying because of the noninvasive, physiologic, and quantifiable properties of the test.

However, numerous factors such as patient status, test meal composition and preparation, image acquisition parameters, and the method of data analysis can affect the accuracy and reproducibility of the results. To reduce variation in test performance and results, a standardized GES protocol was needed. In 2009, the Society of Nuclear Medicine and Molecular Imaging (SNMMI) published Procedure Guideline for Adult Solid-Meal Gastric Emptying Study 3.0 (2). The standardized protocol must be strictly followed to deliver reliable results.

The SNMMI standardized GES protocol contains precise instructions for patient preparation, including medication withholding, blood glucose level, and fasting state; meal ingredients and preparation; radiopharmaceutical dose; image views and frequency; and image processing using the geometric mean, decay correction, and percentage retention (2).

Of specific interest for this study, the requisite GES meal includes $118 \mathrm{~mL}$ (4 oz) of liquid egg whites such as Eggbeaters (ConAgra Foods, Inc.), 2 slices of toasted white bread, $30 \mathrm{~g}$ of jam or jelly, and $120 \mathrm{~mL}$ of water (2). To prepare the meal, $18.5-37 \mathrm{MBq}(0.5-1 \mathrm{mCi})$ of ${ }^{99 \mathrm{~m}} \mathrm{Tc}$-sulfur colloid (SC) is added to the liquid egg whites, which are then beaten well and cooked in a microwave oven or nonstick skillet. The egg whites with the ${ }^{99 \mathrm{~m}} \mathrm{Tc}-\mathrm{SC}$ are stirred once or twice during cooking and cooked until firm. The jelly is spread on the bread after it is toasted. The meal items can be eaten separately or as a sandwich along with consumption of the water. 
Despite the publication of the standardized GES protocol in 2009, a study by Farrell et al. in 2017 found considerable protocol noncompliance (3). Specifically, their research showed that $69.3 \%$ of laboratory protocols do not adhere to the standardized meal. Many laboratories (48.1\%) used whole eggs instead of the obligatory liquid egg whites. Numerous laboratories injected the radiopharmaceutical directly into the egg yolk. Anecdotally, Farrell et al. noted that several laboratories asked patients to bring scrambled eggs with them to their appointment, and the technologist then squirted the ${ }^{99 \mathrm{~m}} \mathrm{Tc}-\mathrm{SC}$ on the previously cooked eggs.

As these findings were unquestionably contrary to the advocated SNMMI GES standardized protocol, this study aimed to document the binding rate of ${ }^{99 \mathrm{~m} T c-S C}$ added to whole eggs before or after microwave cooking.

\section{MATERIALS AND METHODS}

\section{Radiotracer Labeling Method}

Two radiotracer labeling methods were used during the study to prepare the eggs for the GES meal. The same cooking technique was used for both radiolabeling methods. In the first method (precooking labeling), $2.6 \mathrm{MBq}(71 \mu \mathrm{Ci})$ of ${ }^{99 \mathrm{~m}} \mathrm{Tc}$-SC were added to the whole egg and mixed well before microwave cooking. In the second method (postcooking labeling), 3.0 MBq (84-89 $\mu \mathrm{Ci})$ of ${ }^{99 \mathrm{~m}} \mathrm{Tc}-\mathrm{SC}$ were squirted onto the previously cooked eggs.

\section{Cooking Method}

One egg (Eggland's Best, LLC) was transferred from its shell to a $118-\mathrm{mL}$ (4-oz) polystyrene foam cup and beaten for $30 \mathrm{~s}$ until the yolk and egg white were well blended. The beaten egg (with or without ${ }^{99 \mathrm{~m}} \mathrm{Tc}-\mathrm{SC}$ as noted above) was cooked by microwave in the same cup as that used for mixing. The mixture was cooked to a firm consistency on high power for $45 \mathrm{~s}$, with stirring at 15-s intervals.

\section{Simulated Gastric Fluid}

The stomach has 2 digestive processes that were replicated in this study: chemical and mechanical (4). During the chemical process, gastric fluid is produced in the stomach and the $\mathrm{pH}$ is

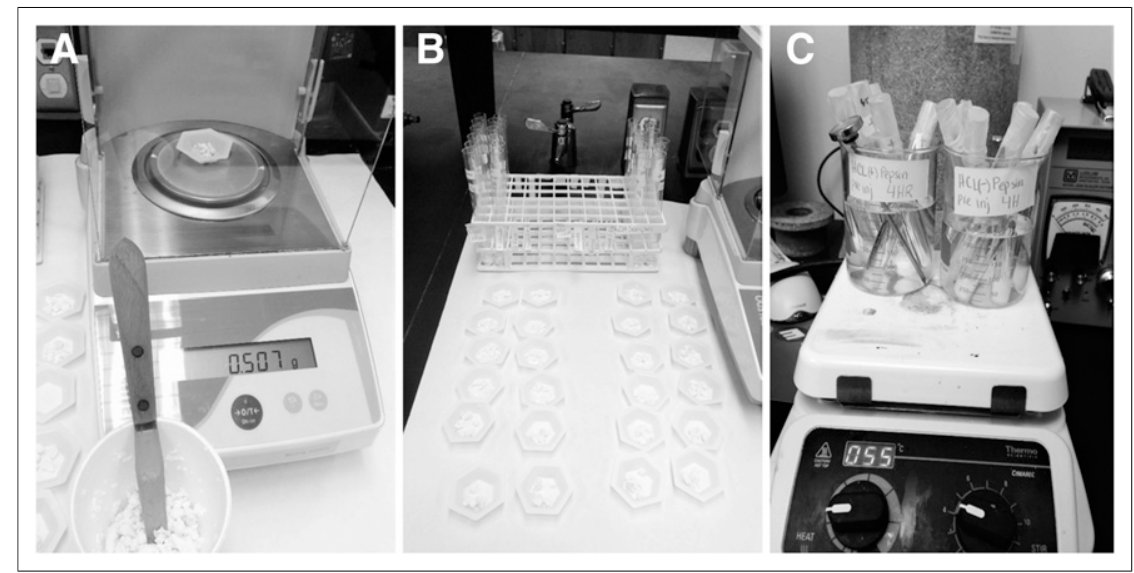

FIGURE 1. Photographs showing weighing method to ensure standardized $0.5-\mathrm{g}$ radiolabeled egg sample size $(A)$, egg samples before being added to simulated gastric fluid (B), and $37^{\circ} \mathrm{C}$ water bath and stirring at $125 \mathrm{rpm}(\mathrm{C})$. between 1.5 and 3.0 (5). Because human gastric fluid was not available for this research, simulated gastric fluid was used to mimic human digestion of proteins. The base of the simulated gastric fluid used in this experiment consisted of $3.5 \mathrm{~mL}$ of hydrochloric acid $(\mathrm{HCl})(0.0847 \mathrm{M})$ combined with $3.06 \mathrm{~g}$ of sodium chloride $(\mathrm{NaCl})(0.1044 \mathrm{M})$ in $500 \mathrm{~mL}$ of distilled water, resulting in a $\mathrm{pH}$ of $1.5(6)$.

In the stomach, $\mathrm{HCl}$ converts pepsinogen, which is also produced in the stomach, to pepsin. Pepsin breaks down proteins to peptides. Several previous studies evaluating the stability of radiotracer binding to the solid-meal component were conducted using only $\mathrm{HCl}$. However, evaluation in $\mathrm{HCl}$ without pepsin may lead to misleading results because the meal may be stable in $\mathrm{HCl}$ but not in $\mathrm{HCl}$ with pepsin (7). For this reason, the study was conducted both without and with $0.1 \mathrm{~g}$ of pepsin added to the test tubes.

\section{Simulated Digestion}

The cooked eggs were cooled to room temperature and chopped to mimic chewing (Fig. 1B). Egg samples (labeled "pre-microwave cooking" and "post-microwave cooking") weighing $0.5 \mathrm{~g}$ (Fig. 1A) were placed in 47 test tubes ( 6 per condition; however, 1 tube was damaged during the experiment and removed) containing $1.25 \mathrm{~mL}$ of $\mathrm{HCl}$ (Fig. 2). Pepsin was combined with HCL for half the test tubes. The tubes were then paraffin-capped and placed in a water bath at $37^{\circ} \mathrm{C}$ with stirrers at $125 \mathrm{rpm}$ for 2 and $4 \mathrm{~h}$ to mimic mechanical and chemical digestion (Fig. 1C).

\section{Radiolabeling Stability Testing}

To separate the sold egg material from the liquid, a filter was created using a 3-mL syringe barrel and sterile gauze. The gauze was cut and folded into $2.54 \times 2.54 \mathrm{~cm}(1 \times 1$ in $)$ sections and packed into the barrel of the syringe (Fig. 3A). The gauze filter was then wet with $0.5 \mathrm{~mL}$ of $1 \%$ bovine serum albumin in isotonic saline. The bovine serum albumin was used to prevent ${ }^{99 \mathrm{~m}} \mathrm{Tc}-\mathrm{SC}$ adhesion to the gauze (8). The bovine serum albumin rinse was followed by a $1-\mathrm{mL}$ isotonic saline rinse.

To separate the solid material from the liquid in the test tubes, the egg and fluid were transferred to the previously prepared syringes with gauze at 2 and $4 \mathrm{~h}$ after the start of the test (Figs. 3B and $3 \mathrm{C}$ ). To ensure that all material was transferred from the test tube to the syringe, the test tube was rinsed once with $1 \mathrm{~mL}$ of isotonic saline, which was also filtered through the syringe.

The gauze with undigested egg material, the liquid filtered from the digestive solution, and the rinse were counted in a well counter ( 8 ). The counted activity was background- and decay-corrected.

Labeling efficiency $(\%)$ was determined as gauze + egg counts (disintegrations per minute)/total counts (disintegrations per minute) $($ gauze + egg + filtrate + rinse $) \times 100$. Independent Student $t$ tests were used to compare the labeling efficiency of the samples. Comparisons were made between labeling methods (precooking labeling vs. postcooking labeling), between incubation times ( 2 vs. $4 \mathrm{~h}$ ), and between $\mathrm{HCl}$ with and without added pepsin. 


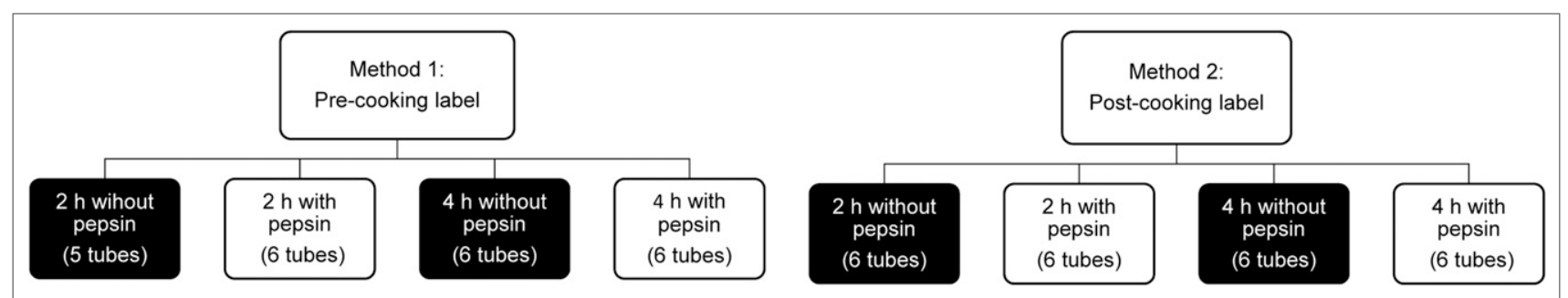

FIGURE 2. Egg labeling test conditions. Two methods were used to radiolabel whole eggs with $99 \mathrm{mTC}$ TC. In method 1 , $99 \mathrm{mTC}$-SC was added to beaten eggs before microwave cooking. In method 2, 99mTc-SC was squirted on eggs after microwave cooking. Stability of radiolabel was tested for both methods at 2 and $4 \mathrm{~h}$. Stability was also tested in gastric fluid simulated using just $\mathrm{HCl}$ and using HCL with pepsin.

\section{RESULTS}

Comparing the timing of the ${ }^{99 \mathrm{~m}} \mathrm{Tc}-\mathrm{SC}$ egg labelingwhether the tracer was added before or after microwave cooking - showed that no matter whether the sample was counted at $2 \mathrm{~h}$ or $4 \mathrm{~h}$ and with or without pepsin added to the digestive solution, the radiotracer binding to the eggs was always significantly higher when the radiotracer was added before the eggs were cooked. For example, in the plain $\mathrm{HCl}$ solution at $2 \mathrm{~h}$, the labeling efficiency was $95.4 \%$ when the radiotracer was added before cooking and $42.3 \%$ when added after cooking $(P<0.001)$. Similarly, in the digestive fluid with pepsin at $2 \mathrm{~h}$, the labeling was significantly higher, at $73.3 \%$, when the radiotracer was added before cooking than the $43.3 \%$ when added after cooking $(P<0.001)$ (Tables $1-3)$.

Comparing the stability of the radiotracer labeling over time showed that for simulated digestive fluid with just $\mathrm{HCl}$, when the radiotracer was added before cooking, the labeling efficiency of the eggs significantly decreased between $2 \mathrm{~h}(95.4 \%)$ and $4 \mathrm{~h}(78.0 \%)(P=0.01)$. Similarly, when the radiotracer was added after cooking, the labeling efficiency also significantly decreased at $2 \mathrm{~h}(57.3 \%)$ and
$4 \mathrm{~h}(39.5 \%)(P=0.02)$. However, when the digestive fluid contained both $\mathrm{HCl}$ and pepsin, radiotracer labeling efficiency did not change significantly between $2 \mathrm{~h}$ and $4 \mathrm{~h}$ no matter whether the radiotracer was added before $(73.3 \%$ vs. $73.1 \%, P=0.94)$ or after cooking $(43.3 \%$ vs. $35.0 \%$, $P=0.10)$.

\section{DISCUSSION}

The accuracy and reproducibility of nuclear medicine GES require strict adherence to the SNMMI standardized protocol (1). A recent study by Farrell et al. found that a majority of nuclear laboratories performing GES are not in compliance with the standardized protocol (3). In particular, almost half the laboratories evaluated used whole eggs in the test meal as opposed to the requisite liquid egg whites. In addition, Farrell et al. noted that several laboratories attempted to label the eggs by adding the radiotracer after cooking. Both methods are contrary to the methods detailed in the SNMMI standardized protocol.

This study aimed to document the labeling efficiency of ${ }^{99 \mathrm{~m}} \mathrm{Tc}-\mathrm{SC}$ added to whole eggs before and after microwave cooking. The experiment showed that no matter what the test condition, radiolabeling efficiency was significantly higher when ${ }^{99 \mathrm{~m}} \mathrm{Tc}-$ $\mathrm{SC}$ was added to eggs before cooking than when ${ }^{99 \mathrm{~m} T c-S C}$ was squirted on eggs after cooking. This finding persisted over time, with the precooking method still showing significantly higher radiolabeling at 2 and $4 \mathrm{~h}$ after the egg was placed in the simulated gastric fluid.

The study finding that labeling efficiency was significantly higher when ${ }^{99 \mathrm{~m}} \mathrm{Tc}-\mathrm{SC}$ was added to eggs before cooking was not surprising. It is known that ${ }^{99 \mathrm{~m}} \mathrm{Tc}-\mathrm{SC}$ fixes to albumin (egg white protein), which denatures as it cooks, binding the ${ }^{99 \mathrm{~m}} \mathrm{Tc}-\mathrm{SC}$. Adding ${ }^{99 \mathrm{~m}} \mathrm{Tc}-\mathrm{SC}$ to previously cooked eggs does not allow the ${ }^{99 \mathrm{~m}} \mathrm{Tc}-\mathrm{SC}$ to bind to the albumin and then be denatured (10). The radiolabeling range of $35.0 \%-56.3 \%$
FIGURE 3. (A) To test stability of radiolabeled eggs, filter was created using gauze and $3-\mathrm{mL}$ syringe. Gauze was wet with $0.5 \mathrm{~mL}$ of $1 \%$ bovine serum albumin to prevent

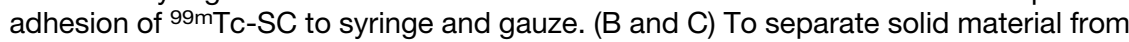
liquid in test tubes, egg and digesting fluid were transferred to previously prepared syringes with gauze at $2 \mathrm{~h} \mathrm{(B)}$ and $4 \mathrm{~h}(\mathrm{C})$ after start of test. 
TABLE 1

Percentage of ${ }^{99 m T c-S C}$ Bound to Whole Eggs Before and After Cooking

\begin{tabular}{clll}
\hline Parameter & Before cooking & After cooking & $P$ \\
\hline No pepsin & & & \\
2-h incubation & $95.4 \%( \pm 2.4 \%)$ & $57.3 \%( \pm 8.4 \%)$ & $<0.001$ \\
4-h incubation & $78.0 \%( \pm 11.5 \%)$ & $39.5 \%( \pm 10.4 \%)$ & $<0.001$ \\
Pepsin & & & \\
2-h incubation & $73.3 \%( \pm 10.9 \%)$ & $43.3 \%( \pm 8.8 \%)$ & $<0.001$ \\
4-h incubation & $73.1 \%( \pm 8.7 \%)$ & $35.0 \%( \pm 10.7 \%)$ & $<0.001$ \\
\hline
\end{tabular}

TABLE 2

Percentage of ${ }^{99 m T C-S C ~ B o u n d ~ t o ~ W h o l e ~ E g g s ~ a t ~ 2-~ a n d ~}$ 4-Hour Incubations

\begin{tabular}{clll}
\hline Parameter & 2-h incubation & 4-h incubation & $P$ \\
\hline Before cooking & & & \\
No pepsin & $95.4 \%( \pm 2.4 \%)$ & $78.0 \%( \pm 11.5 \%)$ & 0.014 \\
Pepsin & $73.3 \%( \pm 10.9 \%)$ & $73.1 \%( \pm 8.7 \%)$ & 0.941 \\
After cooking & & & \\
No pepsin & $57.3 \%( \pm 8.4 \%)$ & $39.5 \%( \pm 10.4 \%)$ & 0.019 \\
Pepsin & $43.3 \%( \pm 8.8 \%)$ & $35.0 \%( \pm 10.7 \%)$ & 0.097 \\
\hline
\end{tabular}

TABLE 3

Percentage of ${ }^{99 m T c}$-SC Bound to Whole Eggs Without and With Pepsin

\begin{tabular}{cccc}
\hline Parameter & No pepsin & Pepsin & $P$ \\
\hline Before cooking & & & \\
2-h incubation & $95.4 \%( \pm 2.4 \%)$ & $73.3 \%( \pm 10.9 \%)$ & 0.003 \\
4-h incubation & $78.0 \%( \pm 11.5 \%)$ & $73.1 .0 \%( \pm 8.7 \%)$ & 0.424 \\
After cooking & & & \\
2-h incubation & $57.3 \%( \pm 8.4 \%)$ & $43.3 \%( \pm 8.8 \%)$ & 0.018 \\
4-h incubation & $39.5 \%( \pm 10.4 \%)$ & $35.0 \%( \pm 10.7 \%)$ & 0.480 \\
\hline
\end{tabular}

in the postcooking method was expected. Thus, the postcooking method of radiolabeling, in which the ${ }^{99 \mathrm{~m} T c-S C}$ is squirted on eggs after microwave cooking, is not optimal and should not be used.

The results of this study further showed that when egg labeling efficiency was tested in a simulated gastric fluid containing only $\mathrm{HCl}$, the labeling was less stable and the labeling efficiency decreased significantly between 2 and $4 \mathrm{~h}$. This finding occurred for both radiolabeling cooking methods. However, when the simulated digestive fluid more closely mimicked real human digestive fluid including pepsin, the labeling efficiency remained unchanged between 2 and $4 \mathrm{~h}$. This finding indicates that use of a simulated gastric fluid with only $\mathrm{HCl}$ may be misleading in that the radiolabeled eggs may not be as stable in $\mathrm{HCl}$ as in $\mathrm{HCl}$ with pepsin (7).

The results of this study are similar to the findings obtained by Knight et al. in 2007 (8). At 2 h, Knight et al. found the labeling efficiency of whole eggs tested in $\mathrm{HCl}$ (precooking labeling) to be $96.9 \%$, compared with our finding of $95.4 \%$. In a similar experiment, Ertay et al. (9) documented a labeling efficiency of $95 \%$. In human gastric fluid at $2 \mathrm{~h}$ (9), the labeling efficiency obtained by Ertay et al. was $73.1 \%$. The labeling efficiency in this experiment using simulated gastric fluid was $73.3 \%$.
Incongruently, Knight et al. had different results at $4 \mathrm{~h}$ in $\mathrm{HCl}$ testing fluid without pepsin (8). In their experiment, the labeling efficiency was not significantly different between $2 \mathrm{~h}$ and $4 \mathrm{~h}$. The labeling efficiency was $96.9 \%$ at $2 \mathrm{~h}$ and $97.3 \%$ at $4 \mathrm{~h}$. However, in our experiment, the labeling efficiency tested in $\mathrm{HCl}$ decreased significantly from $95.4 \%$ at $2 \mathrm{~h}$ to $78.0 \%$ at $4 \mathrm{~h}$. Contrarily, in human gastric fluid, Knight et al. found that the labeling efficiency decreased significantly from $73.1 \%$ at $2 \mathrm{~h}$ to $42.5 \%$ at $4 \mathrm{~h}$. However, our results found that the labeling efficiency remained unchanged, at $73.3 \%$ at $2 \mathrm{~h}$ and $73.1 \%$ at $4 \mathrm{~h}$.

The reason for the incongruent findings at $4 \mathrm{~h}$ between our results and those of Knight et al. is difficult to suppose. However, a possible explanation may be that Knight et al. used actual human gastric fluid obtained from subjects undergoing elective endoscopy (8). Our experiment used $\mathrm{HCl}$ with pepsin added to simulate gastric fluid.

Differences in experimental technique related to egg beating and mixing or cooked egg firmness may also explain the difference in results at $4 \mathrm{~h}$ between our experiment and that of Knight et al. (8). Differences in binding stability could have been exacerbated by the use of whole eggs as opposed to the recommended egg whites. ${ }^{99 \mathrm{~m} T c-S C}$ binds to the albumin or egg white protein and the egg white is denatured and bound during cooking (10). Knight et al. demonstrated the possible fluctuation while microwaving whole eggs, which, unlike the liquid egg whites, allowed the albumin to separate from the yolks during the cooking process. ${ }^{99 \mathrm{~m}} \mathrm{Tc}-\mathrm{SC}$ does not bind to egg yolks.

Strict adherence to the standard gastric emptying meal and preparation method is critical for accurate GES results. The ${ }^{99 \mathrm{~m}} \mathrm{Tc}-\mathrm{SC}$ must bind tightly to the egg, forming the solid component of the meal (11). The gastric emptying rate of solids and liquids is different. Liquids begin leaving the stomach immediately on ingestion, with an exponential rate of emptying. Solids enter the stomach and are broken down by mechanical contraction and gastric fluid. Solids empty more slowly after a lag phase and then at a fixed rate. If the ${ }^{99 \mathrm{~m} T c-S C}$ is not tightly bound to the eggs (solid component) because of improper labeling, the study essentially becomes a liquid GES because part of the meal exits the stomach as a liquid. This creates the possibility of a false-negative GES.

This study was somewhat limited because whole eggs instead of the recommended liquid egg whites were used to test radiolabeling stability. However, considering the findings by Farrell et al. (3) that many laboratories use whole eggs and add the radiotracer after cooking, the results of this study using whole eggs and different cooking methods demonstrates unsatisfactory radiolabeling efficiency. This study is also limited in that the adequacy of mixing and cooking was not tested. However, Knight et al. (8) imaged the cooking methods of whole eggs versus liquid egg whites and noted nonhomogeneous albumin and yolk during microwave cooking. Bonta et al. evaluated the 
quality of meal preparation and egg mixing between technologists by imaging the radiolabeled eggs before patient ingestion (12).

\section{CONCLUSION}

The accuracy and reproducibility of nuclear medicine GES are dependent on strict compliance with the SNMMI standardized protocol. This study documented the radiolabeling stability of ${ }^{99 \mathrm{~m}} \mathrm{Tc}-\mathrm{SC}$ added to whole eggs before and after microwave cooking. The results showed that the addition of ${ }^{99} \mathrm{~m}$ Tc-SC to whole eggs after cooking resulted in inferior binding of the radiotracer to the eggs, with the binding deteriorating significantly over time. The study further demonstrated that the results of radiolabeling efficiency vary depending on whether $\mathrm{HCl}$ or $\mathrm{HCl}$ with pepsin is used to simulate gastric fluid. Radiolabeling stability decreased over time when $\mathrm{HCl}$ without pepsin was used. The results emphasize the criticality of adherence to the standardized meal and preparation, as alternate cooking methods have varying radiolabeling efficiencies.

\section{DISCLOSURE}

No potential conflict of interest relevant to this article was reported.

\section{ACKNOWLEDGMENTS}

We had access to the laboratories and supplies of Baptist College Health Sciences, with Charity Brannen, PhD, Assistant Professor of Chemistry, and to those of UT Health Sciences, with Vivian S. Loveless, PharmD, BCNP, FAPhA, Associate Professor of Pharmaceutical Sciences. Robert Irwin, DPh, GE Nuclear Pharmacy, and Mohammed
Moinuddin, MD, were also a part of our supporting staff for any questions or concerns that arose during this research project.

\section{REFERENCES}

1. Abell TL, Camilleri M, Donohoe K, et al. Consensus recommendations for gastric emptying scintigraphy: a joint report of the American Neurogastroenterology and Motility Society and the Society of Nuclear Medicine. J Nucl Med Technol. 2008;36:44-54.

2. Donohoe KJ, Maurer AH, Ziessman HA, et al. Procedure guideline for adult solid-meal gastric-emptying study 3.0. J Nucl Med Technol. 2009;37:196-200.

3. Farrell MB, Costello M, McKee JD, Gordon LL, Fig LM. Compliance with gastric-emptying scintigraphy guidelines: an analysis of the Intersocietal Accreditation Commission database. J Nucl Med Technol. 2017;45:6-13.

4. Urbain JLC, Malmud LS. Gastrointestinal system. In: Christian PE, WaterstramRich KM, eds. Nuclear Medicine and PET/CT Technology and Techniques. 6th ed. St. Louis, MO: Mosby Elsevier; 2007:526-532.

5. Vertzoni M, Dressman J, Butler J, Hempenstall J, Reppas C. Simulation of fasting gastric conditions and its importance for the in vivo dissolution of lipophilic compounds. Eur J Pharm Biopharm. 2005;60:413-417.

6. Guzman ML, Marques M, Olivera M, Stippler E. Enzymatic activity in the presence of surfactants commonly used in dissolution media, part 1: pepsin. Results Pharma Sci. 2016;6:15-19.

7. Knight LC. Update on GI radiopharmaceuticals and dosimetry estimates. Semin Nucl Med. 2012;42:138-144.

8. Knight LC, Kantor S, Doma S, Parkman HP, Maurer AH. Egg labeling methods for gastric emptying scintigraphy are not equivalent in producing a stable solid meal. J Nucl Med. 2007;48:1897-1900.

9. Ertay T, Doğan AS, Ulker O, Durak H. In vitro evaluation of Tc-99m radiopharmaceuticals for gastric emptying studies. Mol Imaging Radionucl Ther. 2014; 23:21-24.

10. Yoshinori M, Tatsushi N, Noriyuki H. Thermally induced changes in egg white proteins. J Agric Food Chem. 1990;38:2122-2125.

11. Farrell MB. Gastric emptying scintigraphy. In: Bolus N, Farrell MB, Zimmerman J, eds. Abdominal imaging 2017: Quality Safety, \& Dose Optimization. Reston, VA: Society of Nuclear Medicine and Molecular Imaging; 2017:23-39.

12. Bonta DV, Brandon DC, Hernandez J, Patel M, Grant S, Alazraki N. Clinical intervention for quality improvement of gastric-emptying studies. $\mathrm{J} \mathrm{Nucl} \mathrm{Med}$ Technol. 2014;42:274-277. 